\title{
Comparison of Oral Antidiabetic Drugs as Add-On Treatments in Patients with Type 2 Diabetes Uncontrolled on Metformin: A Network Meta-Analysis
}

\author{
Dan Qian · Tiantian Zhang - Peiying Zheng · Zhuoru Liang • \\ Sen Wang $\cdot$ Jingmei Xie $\cdot$ Lina Zhao $\cdot$ Ying Zhang $\cdot$ Bing Situ
}

Received: June 7, 2018 / Published online: August 18, 2018

(c) The Author(s) 2018

\begin{abstract}
We assessed the efficacy and safety of oral antidiabetic drugs (OADs) as an add-on treatment in patients with type 2 diabetes uncontrolled on metformin. PubMed, the Cochrane Library, and Embase were searched from inception to October 20, 2017. Pairwise and network meta-analyses were conducted using Stata 14.1 software. Odds
\end{abstract}

Dan Qian and Tiantian Zhang contributed equally to this work.

Enhanced Digital Features To view enhanced digital features for this article go to https://doi.org/10.6084/ m9.figshare.6881576.

Electronic supplementary material The online version of this article (https://doi.org/10.1007/s13300018-0482-5) contains supplementary material, which is available to authorized users.

D. Qian · P. Zheng · Y. Zhang · B. Situ $(\bowtie)$

Department of Pharmacy, The Third Affiliated

Hospital of Guangzhou Medical University,

Guangzhou 510150, China

e-mail: lilyst@126.com

T. Zhang $\cdot$ Z. Liang $\cdot$ S. Wang $\cdot$ J. Xie $\cdot$ L. Zhao College of Pharmacy, Jinan University, Guangzhou 510632, China

Y. Zhang ( $\bowtie)$

Department of Endocrinology, The Third Affiliated

Hospital of Guangzhou Medical University,

Guangzhou 510150, China

e-mail: zhangying30412@163.com ratios (ORs) and weighted mean differences (WMDs) were used to evaluate outcomes. Sixtyeight trials including 36,746 patients were analyzed. No significant differences in the risk of major adverse cardiovascular events (MACEs) and all-cause mortality were observed among any class of OADs when combined with metformin. All classes of OADs as add-ons to metformin improved glucose control, while sodium-glucose co-transporter-2 (SGLT-2) inhibitors showed greater fasting plasma glucose (FPG) reductions \{WMD, - 1.49 [95\% confidence interval (CI) -1.69 to -1.28$] \mathrm{mmol} / \mathrm{l}\}$ and $2 \mathrm{~h}$ postprandial glucose (2 h PPG) reductions [WMD, - 3.07 (95\% $\mathrm{CI}-4.12$ to -2.03$) \mathrm{mmol} / \mathrm{l}]$. Thiazolidinediones and sulfonylureas were associated with weight gain [WMD, 2.53 (95\% CI 1.95-3.10) kg and 2.00 (95\% CI 1.63-2.36) kg, respectively] when added to metformin. Sulfonylureas [WMD, 6.52 (95\% CI 4.07-10.45)] were associated with the highest ORs of hypoglycemia. Our results suggest that the seven classes of OADs were not associated with any increased risk of MACEs or all-cause mortality when combined with metformin. Most OADs were associated with similarly large reductions in HbA1c levels when added to metformin, while SGLT-2 inhibitors might be the best option for reducing body weight, FPG, and 2-h PPG.

Keywords: Metformin; Network meta-analysis; Oral antidiabetic drug; Type 2 diabetes mellitus 


\section{INTRODUCTION}

Type 2 diabetes mellitus (T2DM) is a chronic metabolic disorder characterized by insufficient or relatively insufficient insulin secretion, combined with insulin resistance, glucose and lipid metabolism disorders, and various chronic complications $[1,2]$. According to the International Diabetes Federation, at present, 425 million people globally suffer from diabetes; this number will increase to 629 million by 2045 [3].

Metformin is the first treatment choice for T2DM patients, as recommended by the guidelines [4-6]. However, when the disease is prolonged, the insulin function of patients declines, and they often experience chronic complications [7]. Monotherapy or increasing the therapeutic dose of metformin cannot effectively control blood glucose; thus, a combined approach with other oral antidiabetic agents with different mechanisms of action should be adopted. Oral delivery of antidiabetic agents is widely used owing to high patient adherence; the currently approved second-line oral antidiabetic agents include sodium-glucose co-transporter-2 (SGLT-2) inhibitors, dipeptidyl peptidase 4 (DPP-4) inhibitors, thiazolidinediones (TZDs), sulfonylureas (SUs), alpha-glucosidase inhibitors (AGIs), and meglitinides. Various factors contribute to the selection of second-line oral antidiabetic drugs (OADs). Besides hypoglycemic effects, it is important to consider the incidence of cardiovascular events and mortality. To provide the best treatment to patients, clinicians must balance patient compliance, glucose-lowering efficacy, drug-to-drug interactions, and the side-effect profiles of the above mentioned hypoglycemic agents.

In contrast to traditional meta-analyses which rely on direct comparisons and fail to assess the relative effects of various interventions simultaneously [8], a network meta-analysis (NMA) can be used to evaluate multiple direct or indirect interventions and to quantify and sort the efficacy and safety of each of these measures, so as to screen the most effective and tolerable interventions [9]. To provide a more complete profile of the efficacy and safety of OADs in patients with T2DM uncontrolled on metformin, we conducted a systematic review and network meta-analysis.

\section{METHODS}

\section{Search Strategy}

We searched multiple databases, including PubMed, the Cochrane Library, and Embase, for randomized clinical trials (RCTs) from inception to October 20, 2017. Search terms included "SGLT-2 inhibitor," "DPP-4 inhibitor," "SU," "TZD," "AGI," "meglitinide," "diabetes mellitus," and "RCT." Detailed search strategies are listed in Table S1 of the Electronic supplementary material (ESM). Additional reference lists from review articles and ClinicalTrials.gov were also searched to identify published and unpublished trials. No language restrictions were applied.

\section{Study Selection and Data Extraction}

Eligible studies had to meet the following criteria: (1) RCTs with full-text publication; (2) patients with T2DM inadequately controlled on metformin monotherapy (initial metformin dose: $\geq 1500 \mathrm{mg} /$ day $\quad$ or $1000 \mathrm{mg} /$ day); (3) study duration $\geq 24$ weeks; (4) the drug classes targeted in our comparison were SGLT-2 inhibitors, DPP-4 inhibitors, SUs, TZDs, AGIs, metformin (high-dose metformin, $\geq 2000 \mathrm{mg} /$ day), and meglitinides; (5) at least one of our selected outcomes was presented in the included published articles. Efficacy outcomes included glycated hemoglobin (HbA1c), fasting plasma glucose (FPG), 2-h postprandial glucose (2-h PPG), and body weight. Safety outcomes included major adverse cardiovascular events (MACEs), all-cause mortality, hypoglycemia, serious adverse events (SAEs), urinary tract infection (UTI), and diarrhea. MACE comprised cardiovascular death, nonfatal myocardial infarction, and nonfatal stroke. The following patients were excluded: (1) pregnant patients; (2) children ( $\leq 18$ years); (3) patients with a severe cardiovascular disease or inadequate liver and renal function. 
Two authors (D.Q. and T.Z.) independently screened references and extracted data using a predefined data collection form including first author (publication year); trial registration number; interventions; sample size; follow-up duration; mean age; mean sex; mean HbA1c; mean body weight; mean body mass index (BMI); and mean diabetes duration. The third author (P.Z.) resolved any discrepancy. Primary safety outcomes included MACEs and all-cause mortality, and primary efficacy outcomes included HbA1c and body weight. The longest follow-up period was considered for studies with different follow-up durations. Data were extracted from ClinicalTrials.gov if relevant information was not available in the published literature. When both published and unpublished data were available, the published data were used. Ethical approval was not required for this meta-analysis.

\section{Risk of Bias}

Study quality was evaluated by two authors (D.Q. and P.Z.) based on the Cochrane risk-ofbias tool [10]. The assessment details included random sequence generation, allocation concealment, blinding, missing outcome data, and selective reporting.

\section{Statistical Analysis}

Pairwise analyses and NMAs were conducted to calculate odds ratios (ORs) for dichotomous variables (MACEs, all-cause mortality, SAEs, hypoglycemia, UTI, and diarrhea) and weighted mean differences (WMDs) for continuous variables (HbA1c, body weight, FPG, and 2-h PPG), together with the corresponding 95\% confidence intervals (CIs).

For conventional pairwise meta-analysis, a DerSimonian-Laird random effects model was used [11]. Statistical heterogeneity was measured using the $I^{2}$ statistic, and was judged as low $(<25 \%)$, moderate $(25-75 \%)$, or high (> 75\%) [12]. For NMA, a frequentist model was used [13]. NMA was performed in Stata version 14.1 using the mvmeta and network commands and programmed Stata routines [14, 15]. The assumption of NMA allows for heterogeneity in intervention effects among studies [16], but does not allow for significant differences in study design. To rank the probabilities of each intervention for various outcomes, we used rankograms, the surface under the cumulative ranking curve (SUCRA), and mean ranks. The SUCRA value reflects intervention effectiveness; the larger the SUCRA value, the better the intervention rank [17]. A 0.5 zero-cell correction was applied before the meta-analysis when studies reported zero events [18].

To check for model inconsistency, a loopspecific approach was used to assess the difference between direct and indirect estimates in each closed triangular or quadrangular loop [19]. The heterogeneity among studies in each closed loop was evaluated using the inconsistency factor (IF). If the 95\% CIs of IF values do not include zero, it shows that the IF direction is important [20]. To check the global heterogeneity in networks, a "design-by-treatment" model was used [21]. Additionally, the absence of small-study effects was tested for using a comparison-adjusted funnel plot [22]. To test the robustness of our results, we performed a sensitivity analysis by excluding single-blinded and unblinded studies on the primary outcomes.

All analyses were conducted using Stata 14.1 software; $P$ values of $<0.05$ were regarded as statistically significant.

\section{Compliance with Ethics Guidelines}

This article is based on previously conducted studies, and did not involve any participation of humans or animals.

\section{RESULTS}

\section{Study Characteristics and Quality Assessment}

The literature search retrieved 12664 articles, 1411 of which were duplicates and were removed. After reviewing the titles and abstracts, 495 eligible studies were included for 


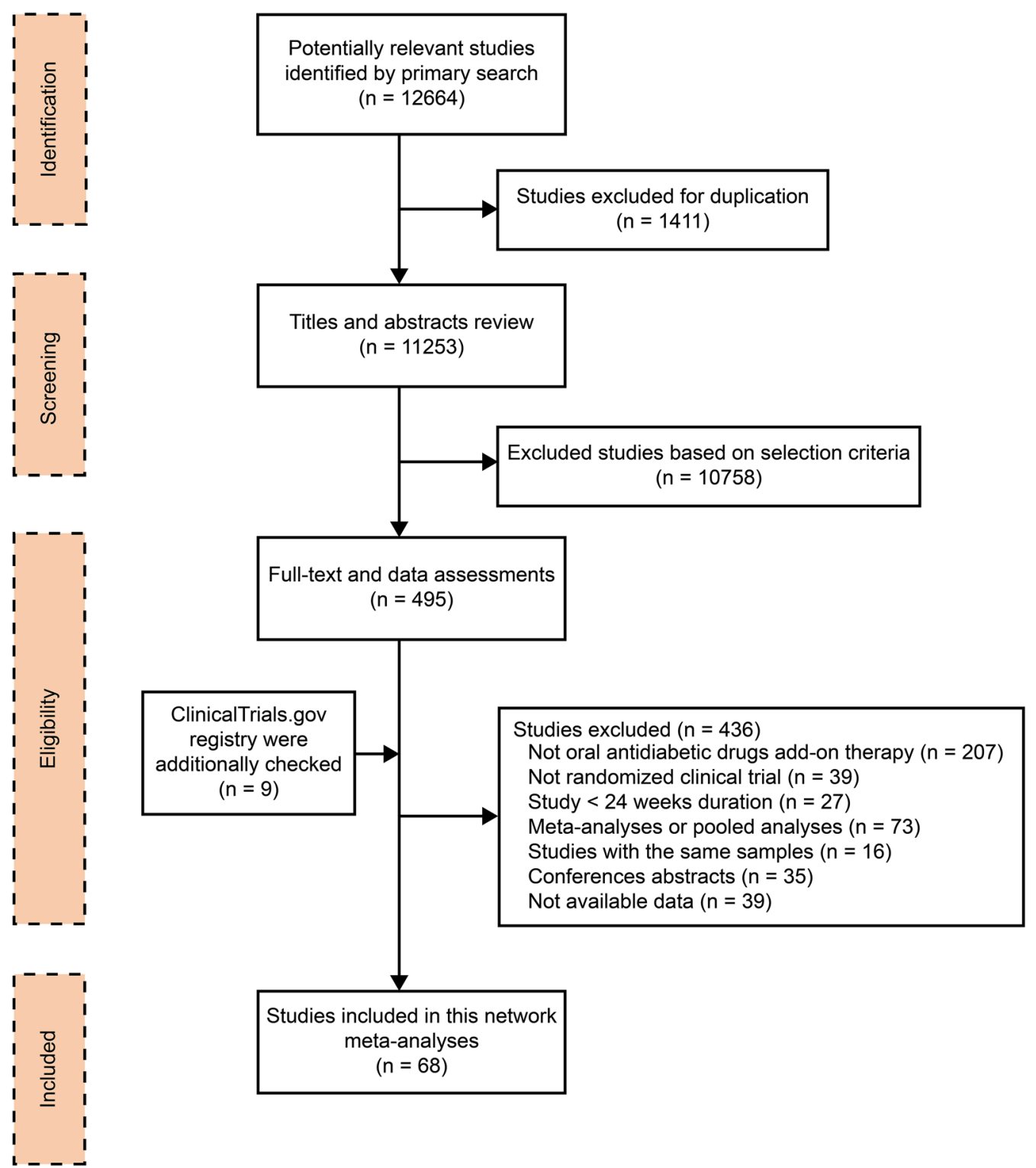

Fig. 1 Flow chart of the search for eligible studies

full-text screening; 68 eligible RCTs involving 36,746 adults fulfilled the inclusion criteria and were included in our NMA (Fig. 1). The involved studies were published from 2000 to 2017 and the follow-up periods ranged from 24 to 208 weeks. The mean patient age in each study was 52-72.7 years; mean baseline $\mathrm{HbA1c}$, 6.9-9.0\%; mean baseline weight, 63.9-99.3 kg; and the mean duration of diagnosed diabetes, 4.1-14.2 years. Table S2 of the ESM provides detailed information on the included trials. The results of a bias risk assessment are shown in
Table S3 of the ESM. Some studies did not provide information on randomization, allocation concealment, and blinding methods.

\section{Network Consistency}

Network maps for all outcomes are graphically displayed in Fig. S1 of the ESM. There was no loop inconsistency between evidence derived from direct and indirect comparisons because the $95 \%$ CIs of IF values included zero, with the 
exception of the direct and indirect comparison in the placebo-DPP4-AGI closed loop for HbA1c (Table S4 of the ESM). Additionally, the designby-treatment model did not detect global inconsistency within any network ( $p$ all $>0.05$, Table S5 of the ESM). Results of pairwise random-effects meta-analyses for all outcomes are displayed in Table S6 of the ESM, and the contribution of each study to the NMA is shown in Table S7 of the ESM.

\section{Meta-Analysis}

\section{Primary Efficacy Outcomes: Glycated Hemoglobin and Body Weight}

Changes in $\mathrm{HbA1c}$ from baseline were reported in 66 RCTs. NMA results demonstrated a mean HbA1c reduction for all drug classes compared with placebo when added to metformin: $-0.73 \%$ (95\% CI -0.84 to -0.62$)$ for SGLT-2 inhibitors; $-0.65 \%$ (95\% CI -0.73 to -0.56$)$ for DPP-4 inhibitors; - 0.69\% (95\% CI -0.80 to -0.57$)$ for SUs; $-0.73 \%(95 \% \mathrm{CI}-0.87$ to $-0.58)$ for TZDs; $-0.75 \%(95 \%$ CI -0.95 to -0.55 ) for AGIs; $-0.49 \%$ (95\% CI -0.70 to -0.28 ) for high-dose metformin; and $-0.53 \%$ (95\% CI -0.94 to -0.11$)$ for meglitinides (Table 1, Fig. S2 of the ESM). There were no significant differences in HbA1c level reduction between any class of OADs when added to metformin, with the exception of the comparison between SGLT-2 inhibitors and high-dose metformin $[-0.24 \% \quad(95 \% \quad$ CI -0.46 to $-0.01)$.

Evidence was available from 46 RCTs for body weight change from baseline across the network. Compared with placebo, NMA showed a significant mean change in body weight for some of the assessed OADs when added to metformin: $-2.23 \mathrm{~kg}(95 \% \mathrm{CI}-2.55$ to -1.91$)$ for SGLT-2 inhibitors; - $0.82 \mathrm{~kg}(95 \% \mathrm{CI}-1.61$ to - 0.04) for AGIs; $2.00 \mathrm{~kg}$ (95\% CI 1.63-2.36) for SUs; and $2.53 \mathrm{~kg}$ (95\% CI 1.95-3.10) for TZD, while no differences were found for the DPP-4 inhibitors [ $-0.11 \mathrm{~kg}$ (95\% CI -0.39 to 0.18$)$ ] and high-dose metformin [- $0.62 \mathrm{~kg}(95 \% \mathrm{CI}$ -1.46 to 0.23 )] (Table 1 , Fig. S2 of the ESM). The SGLT-2 inhibitors reduced body weight to a greater extent than all other OADs.

\section{Secondary Efficacy Outcomes: FPG and 2-h PPG}

Fifty-seven RCTs reported changes in FPG. NMA results revealed that a FPG reduction was brought about by the following agents as opposed to placebo when combined with metformin: $-1.49 \mathrm{mmol} / \mathrm{l} \quad(95 \%$ CI -1.69 to - 1.28) for SGLT-2 inhibitors; $-0.95 \mathrm{mmol} / \mathrm{l}$ (95\% CI -1.10 to -0.79 ) for DPP-4 inhibitors; $-1.11 \mathrm{mmol} / \mathrm{l}(95 \% \mathrm{CI}-1.34$ to -0.89$)$ for SUs; $-1.26 \mathrm{mmol} / \mathrm{l}$ (95\% CI -1.60 to -0.91 for TZDs; $-1.07 \mathrm{mmol} / \mathrm{l}$ (95\% CI -1.42 to - 0.71) for AGIs; - $0.26 \mathrm{mmol} / \mathrm{l}$ (95\% CI - 0.71 to 0.18 ) for high-dose metformin; and - $0.93 \mathrm{mmol} / \mathrm{l}(95 \% \mathrm{CI}-1.70$ to -0.15$)$ for meglitinides. Among all drug classes, SGLT-2 inhibitors showed greater FPG reductions than other OADs, while TZDs [- $0.23 \mathrm{mmol} / 1$ (95\% $\mathrm{CI}-0.60$ to 0.13$)]$ and meglitinide [ $-0.56 \mathrm{mmol} / \mathrm{l} \quad(95 \%$ CI -1.35 to 0.22$)]$ showed no significant difference in FPG levels (Table 1, Fig. S2 of the ESM).

Levels of 2-h PPG were known from 16 RCTs. NMA results showed a mean 2-h PPG reduction for all drug classes compared with placebo when added to metformin: $-3.07 \mathrm{mmol} / 1$ (95\% CI -4.12 to -2.03$)$ for SGLT-2 inhibitors; $-1.71 \mathrm{mmol} / \mathrm{l}(95 \% \mathrm{CI}-2.26$ to -1.15$)$ for DPP-4 inhibitors; $-1.62 \mathrm{mmol} / \mathrm{l} \quad(95 \%$ CI -2.68 to -0.56$)$ for SUs; $-1.95 \mathrm{mmol} / \mathrm{l}(95 \%$ CI -3.70 to -0.20$)$ for TZDs; $-2.19 \%$ (95\% CI -3.37 to -1.01 ) for AGIs; and $-1.50 \mathrm{mmol} / \mathrm{l}$ (95\% CI -2.99 to -0.01$)$ for high-dose metformin (Table 1, Fig. S2 of the ESM). Among all drug classes, the SGLT-2 inhibitor reduced 2-h PPG to a greater extent than the DPP-4 inhibitor [- $1.37 \mathrm{mmol} / \mathrm{l}(95 \% \mathrm{CI}-2.42$ to -0.32$)]$ and $\mathrm{SU}[-1.45 \mathrm{mmol} / \mathrm{l}(95 \% \mathrm{CI}-2.84$ to -0.07$)]$.

\section{Primary Safety Outcomes: MACEs and All- Cause Mortality}

MACE data were available for 33 RCTs involving 23,168 patients. NMA results revealed that there was no statistically significant difference in the OR of MACEs between any of the classes of OADs when they were added to metformin. Data on all-cause mortality were available from 36 RCTs involving 29,373 patients. There was no association between any class of OADs and 


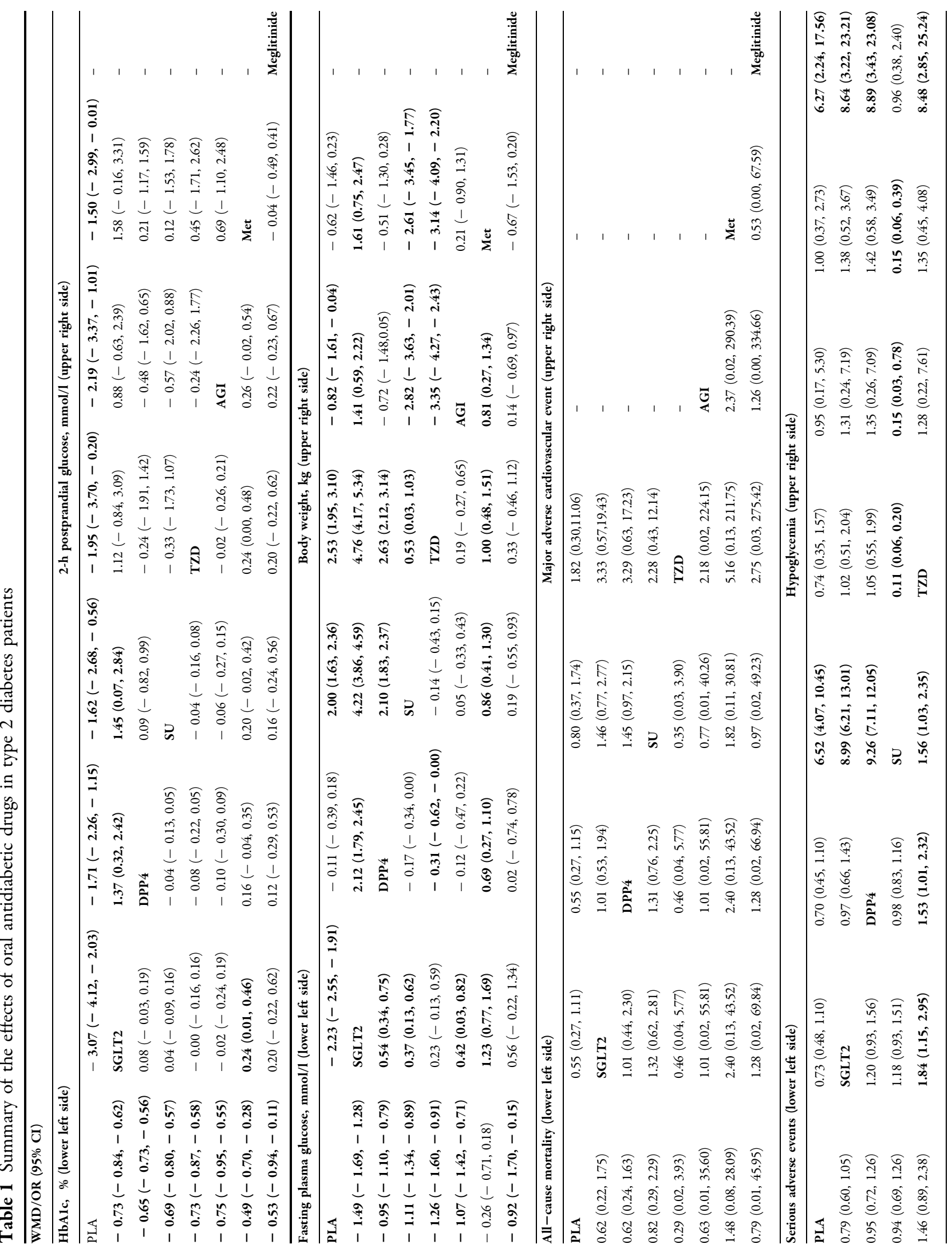




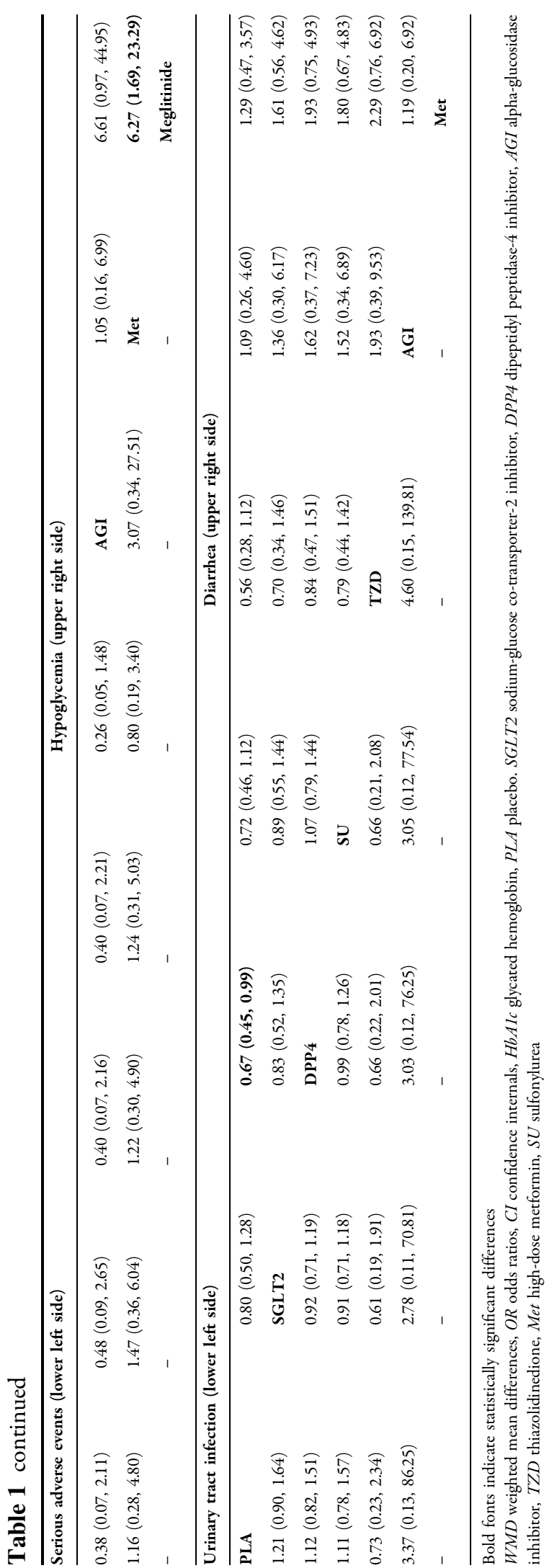

the OR of all-cause mortality (Table 1, Fig. S2 of the ESM).

\section{Secondary Safety Outcomes: Hypoglycemia, SAEs, UTI and Diarrhea}

Evidence on hypoglycemia events was available from 48 RCTs. NMA results showed an increased hypoglycemia risk compared with placebo when added to metformin for some of the studied drugs: [OR, 6.52 (95\% CI 4.07-10.45)] for SUs and [OR, 6.27 (95\% CI 2.24-17.56)] for meglitinides. Compared with SUs, all classes of OADs were associated with a lower hypoglycemia risk, with the exception of meglitinides [OR, 0.96 (95\% CI 0.38-2.40)], which showed no significant difference in hypoglycemia risk (Table 1, Fig. S2 of the ESM).

Compared with placebo, NMA showed no statistically significant difference in the ORs of SAEs, UTI, or diarrhea, with the exception of a lower OR for diarrhea for DPP-4 inhibitors [odds ratio (OR), 0.67 (95\% CI 0.45-0.99)], when it was added to metformin. Compared with TZDs, SGLT-2 inhibitors [OR, $0.54 \quad(95 \% \quad \mathrm{CI}$ 0.34-0.87)], DPP-4 inhibitors [OR, 0.65 (95\% CI 0.43-0.99)], and SUs [OR, $0.64 \quad(95 \%$ CI 0.43-0.97)] were associated with a significantly lower SAE risk. No significant differences in the ORs of UTI or diarrhea were observed between any of the drug classes when they were added to metformin (Table 1, Fig. S2 of the ESM).

\section{Ranking of Interventions and Cluster Analysis}

As shown in Table 2, the SUCRA results revealed that in terms of efficacy aspects, including body weight, FPG, and 2-h PPG, the SUCRA value was highest with SGLT-2 inhibitors + metformin (body weight: 100\%; FPG: 97.3\%; 2-h PPG: 95.1\%). For HbA1c, the SUCRA value was highest in AGIs (79.3\%). For safety aspects like MACEs and SAEs, TZDs + metformin had the lowest SUCRA value (MACEs: 15.2\%; SAEs: 9.7\%). Regarding all-cause mortality, UTI, and diarrhea, the highest SUCRA value was found in TZDs + metformin (all-cause mortality: 75.1\%; UTI: $75.3 \%$; diarrhea: $83.9 \%$ ). With respect to hypoglycemia, the lowest SUCRA value was for 
Table 2 Results of the surface under the cumulative ranking curve (SUCRA)

\begin{tabular}{|c|c|c|c|c|c|c|c|c|c|c|}
\hline \multirow[t]{2}{*}{ Treatments } & \multicolumn{10}{|c|}{ SUCRA values } \\
\hline & HbAlc & $\begin{array}{l}\text { 2-h } \\
\text { PPG }\end{array}$ & FPG & $\begin{array}{l}\text { Body } \\
\text { weight }\end{array}$ & $\begin{array}{l}\text { All-cause } \\
\text { mortality }\end{array}$ & MACE & Hypoglycemia & SAE & UTI & Diarrhea \\
\hline Placebo & 0.1 & 0.7 & 2.1 & 39.1 & 32.5 & 27.9 & 46.9 & 42 & 68 & 26 \\
\hline SGLT-2 Inhibitor & 77.5 & 95.1 & 97.3 & 100 & 60.1 & 81.5 & 74.1 & 77.6 & 31.5 & 49.2 \\
\hline DPP-4 inhibitor & 45.8 & 45.8 & 39.7 & 48.9 & 60.6 & 82.7 & 77.7 & 48.7 & 47.9 & 73.5 \\
\hline Sulfonylurea & 63.3 & 42 & 62.9 & 16.4 & 40.3 & 42.6 & 6.4 & 50.7 & 52.6 & 62.5 \\
\hline Thiazolidinedione & 76.2 & 59 & 78.7 & 0.3 & 75.1 & 15.2 & 72.1 & 9.7 & 75.3 & 83.9 \\
\hline AGI & 79.3 & 67.4 & 56.7 & 76.4 & 51.4 & $N A^{j}$ & 60.6 & 84.8 & 24.9 & 34 \\
\hline Met & 22.4 & 40.1 & 13.5 & 68.9 & 31.4 & NA & 53.9 & 36.4 & NA & 20.9 \\
\hline Meglitinide & 36.4 & NA & 49.2 & NA & 48.6 & NA & 8.4 & NA & NA & NA \\
\hline
\end{tabular}

Boldface is used to indicate the highest and lowest SUCRA values for each parameter

SUCRA surface under the cumulative ranking curve, $A G I$ alpha-glucosidase inhibitor, HbAlc glycated hemoglobin, Met high-dose metformin, 2-h PPG 2-h postprandial glucose, FPG fasting plasma glucose, $M A C E$ major adverse cardiovascular event, $S A E$ serious adverse event, $U T I$ urinary tract infection, $N A$ not applicable

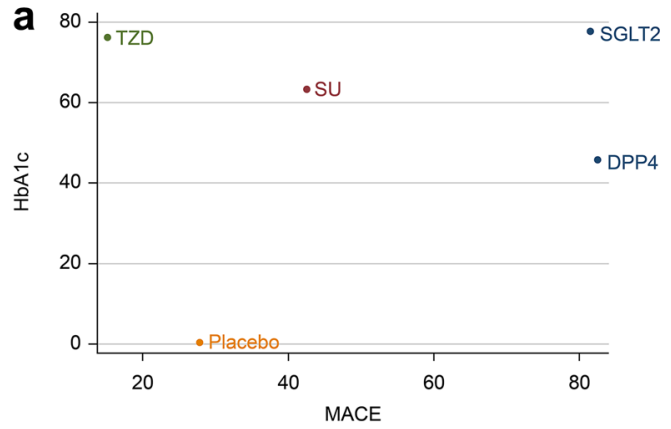

Fig. 2a-b Clustered ranking plots of the network. a MACE ( $x$-axis) and HbAlc ( $y$-axis); b hypoglycemia $(x$-axis) and HbA1c ( $y$-axis). MACE major adverse cardiovascular event, SGLT2 sodium-glucose co-

SUs + metformin (6.4\%), while DPP- $4+$ metformin had the highest SUCRA value (77.7\%). The results of the cluster analysis based on SUCRA values is shown in Fig. 2, and interventions with the same color belong to the same cluster. Treatments located in the upper right corner were superior to the other treatments. According to the cluster analysis results, SGLT-2 inhibitors were associated with a better effect on efficacy and safety outcomes than the other

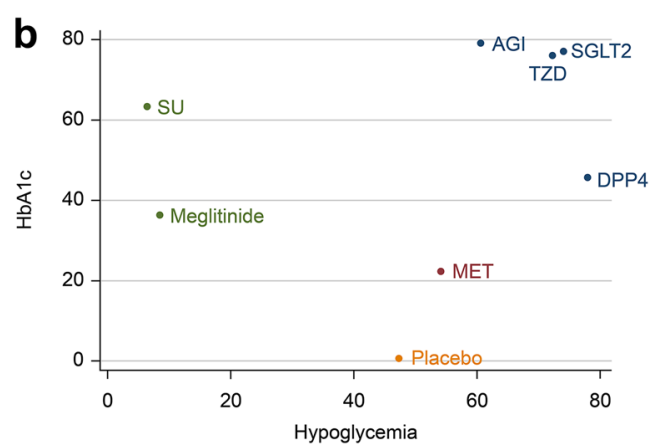

transporter-2 inhibitor, DPP4 dipeptidyl peptidase-4 inhibitor, $A G I$ alpha-glucosidase inhibitor, $T Z D$ thiazolidinedione, $M e t$ high-dose metformin, $S U$ sulfonylurea

studied drugs when the inhibitor was added to metformin.

\section{Sensitivity Analysis and Publication Bias}

In a sensitivity analysis excluding single-blinded and unblinded studies, there was no major change in the primary outcomes (Table 3, Table S8 of the ESM). Comparison-adjusted funnel plots for primary outcomes are shown in 
Table 3 Sensitivity analyses and rank order for the primary outcomes

\begin{tabular}{|c|c|c|c|c|c|c|}
\hline Treatment & Complete analysis & SUCRA & Rank & $\begin{array}{l}\text { Excluding single-blinded } \\
\text { and unblinded studies }\end{array}$ & SUCRA & Rank \\
\hline \multicolumn{7}{|l|}{ MACE } \\
\hline SGLT-2 inhibitor & $0.55(0.27,1.11)$ & 81.5 & 2 & $0.57(0.27,1.22)$ & 77.4 & 2 \\
\hline DPP-4 inhibitor & $0.55(0.27,1.15)$ & 82.7 & 1 & $0.55(0.25,1.20)$ & 84.4 & 1 \\
\hline Sulfonylurea & $0.80(0.37,1.74)$ & 42.6 & 3 & $0.80(0.36,1.80)$ & 43.5 & 3 \\
\hline Thiazolidinedione & $1.82(0.30,11.06)$ & 15.2 & 4 & $1.81(0.29,11.16)$ & 15.7 & 4 \\
\hline \multicolumn{7}{|l|}{ All-cause mortality } \\
\hline SGLT-2 inhibitor & $0.62(0.22,1.75)$ & 60.1 & 3 & $0.65(0.22,1.89)$ & 56.2 & 3 \\
\hline DPP-4 inhibitor & $0.62(0.24,1.63)$ & 60.6 & 2 & $0.62(0.23,1.67)$ & 60.9 & 2 \\
\hline Sulfonylurea & $0.82(0.29,2.29)$ & 40.3 & 6 & $0.82(0.29,2.37)$ & 35.8 & 5 \\
\hline Thiazolidinedione & $0.29(0.02,3.93)$ & 75.1 & 1 & $0.29(0.02,4.00)$ & 74.7 & 1 \\
\hline AGI & $0.63(0.01,35.60)$ & 51.4 & 4 & $0.77(0.04,15.12)$ & 45.1 & 4 \\
\hline Met & $1.48(0.08,28.09)$ & 31.4 & 7 & $\mathrm{NA}^{\mathrm{g}}$ & NA & NA \\
\hline Meglitinide & $0.79(0.01,45.95)$ & 48.6 & 5 & NA & NA & NA \\
\hline \multicolumn{7}{|l|}{ HbAlc } \\
\hline SGLT-2 inhibitor & $-0.73(-0.84,-0.62)$ & 77.5 & 2 & $-0.72(-0.84,-0.60)$ & 77.4 & 2 \\
\hline DPP-4 inhibitor & $-0.65(-0.73,-0.56)$ & 45.8 & 5 & $-0.62(-0.70,-0.53)$ & 43.8 & 5 \\
\hline Sulfonylurea & $-0.69(-0.80,-0.57)$ & 63.3 & 4 & $-0.65(-0.77,-0.54)$ & 57.2 & 4 \\
\hline Thiazolidinedione & $-0.73(-0.87,-0.58)$ & 76.2 & 3 & $-0.65(-0.81,-0.50)$ & 58.2 & 3 \\
\hline AGI & $-0.75(-0.95,-0.55)$ & 79.3 & 1 & $-0.99(-1.26,-0.72)$ & 98.4 & 1 \\
\hline Met & $-0.49(-0.70,-0.28)$ & 22.4 & 7 & $-0.48(-0.81,-0.15)$ & 30.5 & 7 \\
\hline Meglitinide & $-0.53(-0.94,-0.11)$ & 36.4 & 6 & $-0.49(-0.89,-0.10)$ & 34.4 & 6 \\
\hline \multicolumn{7}{|l|}{ Body weight } \\
\hline SGLT-2 inhibitor & $-2.23(-2.55,-1.91)$ & 100 & 1 & $-2.10(-2.46,-1.74)$ & 98.8 & 1 \\
\hline DPP-4 inhibitor & $-0.11(-0.39,0.18)$ & 48.9 & 4 & $-0.07(-0.39,0.25)$ & 55.3 & 3 \\
\hline Sulfonylurea & $2.00(1.63,2.36)$ & 16.4 & 5 & $2.05(1.66,2.44)$ & 19.6 & 4 \\
\hline Thiazolidinedione & $2.53(1.95,3.10)$ & 0.3 & 6 & $2.64(2.01,3.27)$ & 0.4 & 5 \\
\hline AGI & $-0.82(-1.61,-0.04)$ & 76.4 & 2 & $-0.89(-2.30,0.52)$ & 76.8 & 2 \\
\hline Met & $-0.62(-1.46,0.23)$ & 68.9 & 3 & NA & NA & NA \\
\hline
\end{tabular}

SUCRA surface under the cumulative ranking curve, $S G L T-2$ sodium-glucose co-transporter-2 inhibitor, DPP-4 dipeptidyl peptidase-4 inhibitor, $A G I$ alpha-glucosidase inhibitor, Met high-dose metformin, $M A C E$ major adverse cardiovascular event, $N A$ not applicable 
Fig. S3 of the ESM indicating no evidence of a small-study effect in the network.

\section{DISCUSSION}

To provide a comprehensive picture of OADs added to metformin and evidence for clinicians and patients to use during decision-making, we conducted NMA to combine the high-quality data on all the available interventions from the most up-to-date RCTs and simultaneously compare the efficacy and safety of OADs as addon treatments in patients with T2DM uncontrolled on metformin. We found no significant differences in the risk of MACEs and all-cause mortality between any class of OADs used as add-ons to metformin.

Compared with placebo, all classes of OADs improved glucose control (by decreasing HbA1c, FPG, and 2-h PPG levels) when added to metformin. With the exception of high-dose metformin, which achieved significantly higher HbA1c values than SGLT-2 inhibitors, all classes of OADs were associated with similarly large reductions in HbA1c levels when added to metformin. Compared with DPP-4 inhibitors, SUs, and AGIs combined with metformin or high-dose metformin, SGLT-2 inhibitors as an add-on to metformin were associated with the greatest reductions in FPG levels, whereas highdose metformin ranked the lowest for HbA1c, FPG, and 2-h PPG reduction. Among the addons to metformin, SUs and TZDs were associated with more weight gain, while SGLT-2 inhibitors resulted in the largest weight loss. When used as add-ons to metformin, SGLT-2 and DPP-4 inhibitors were associated with infrequent hypoglycemia, while SUs had a higher hypoglycemia risk and were ranked the worst.

The cardiovascular safety of antidiabetic medications has been the focus of recent studies because T2DM patients have been at increased risk of cardiovascular events and mortality $[23,24]$. Consistent with the meta-analysis conducted by Palmer et al. [25], our NMA findings demonstrated that there was no statistical difference in the risk of MACEs or all-cause mortality between any of the classes of OADs when added to metformin. Contrastingly, previous meta-analyses conducted by Wu et al. [26] and Saad et al. [27] demonstrated that SGLT-2 inhibitors had a favorable effect on cardiovascular events compared with placebo and other OADs, including DPP-4 inhibitors, SUs, and TZDs. However, those favorable results were heavily affected in the EMPA-REG OUTCOME trial [28] (NCT01131676) in which no clear differences were found for other SGLT-2 inhibitors, including canagliflozin, dapagliflozin, and ertugliflozin. In that trial, adding empagliflozin to the standard of care significantly reduced the risk of MACEs by $14 \%$, cardiovascular death by $38 \%$, and all-cause mortality by $32 \%$ versus placebo [28]. After excluding the EMPA-REG OUTCOME trial, Tang et al. [29] found no significant difference in the risk of MACEs and all-cause mortality between SGLT-2 inhibitors and placebo. We also did not include that trial in our analysis because it did not focus on dual-therapy regimens with metformin; therefore, no cardiovascular benefit was observed with SGLT-2 inhibitors. Currently, empagliflozin is recommended in the 2017 American Diabetes Association (ADA) guidelines as an add-on therapy for patients with established cardiovascular disease after exhibiting a cardiovascular benefit in RCTs [30]. A recent study demonstrated that patients treated with canagliflozin had a lower risk of cardiovascular events than those who received a placebo, while cardiovascular outcome data for dapagliflozin were insufficient [31]. Ongoing cardiovascular outcome trials with canagliflozin [32], dapagliflozin [33], and ertugliflozin [34] are warranted to assess whether the benefits of cardiovascular events are a class effect or a specific drug effect, and future studies on cardiovascular safety after SGLT-2 inhibitor addition to metformin should be prioritized.

When endogenous glucagon-like peptide-1 (GLP-1) and glucose-dependent insulinotropic polypeptide (GIP) levels are increased, DPP-4 inhibitors promote insulin release from islet $\beta$ cells and inhibit glucagon secretion from islet $\alpha$ cells to decrease the blood glucose level [35]. It has been reported that DPP-4Is might exert some of their effects on insulin secretion by preserving intact GLP-1 and GIP secreted from 
alpha cells, although the insulinotropic effect of GIP has been shown to be reduced in patients with type 2 diabetes [36-38]. Several studies have found no definitive evidence supporting the cardiovascular benefits of DPP-4 inhibitors or TZDs [39-43]. One large-scale study directly compared the effects of pioglitazone and SUs as add-ons to metformin on cardiovascular outcomes, and no significant differences in nonfatal myocardial infarction, nonfatal stroke, and all-cause mortality were observed between them [44]. These findings were consistent with our study results. An ongoing study entitled "Cardiovascular outcomes in participants with type 2 diabetes mellitus" will provide further insights into the cardiovascular outcomes of SGLT-2 inhibitors, DPP-4 inhibitors, SUs, and TZDs [45].

There is a close relationship between obesity and cardiovascular risk. Being overweight may aggravate insulin resistance and increase cardiovascular risk and mortality among T2DM patients $[46,47]$. Therefore, reducing weight is a crucial way to reduce diabetic patient mortality. Additionally, weight loss improves $\beta$-cell function, possibly by reducing insulin resistance, and enhances glycemic control [48]. Patients in this study were overweight, with an average BMI of $30.9 \mathrm{~kg} / \mathrm{m}^{2}$. Hence, clinicians should carefully consider the effects of antidiabetic agents on body weight when making treatment decisions; in particular, for overweight or obese diabetic patients, antidiabetic agents such as SUs and TZDs need to be avoided.

Compared with the therapeutic plan of dual therapy with metformin applied in previous studies [25, 26], our research incorporated studies involving the use of second-line OADs in cases where there was poor glycemic control when metformin was used as a first-line drug; this indicated better control of clinical heterogeneity and a more targeted conclusion. We analyzed the influence of OADs on MACEs, FPG level, 2-hPPG level, UTI, and diarrhea; these parameters were not evaluated in previous studies. However, our study has several limitations. First, in accordance with the American Diabetes Association for the Study of Diabetes position statement, we evaluated each class of OADs as a whole, even though there were some within-class differences [49]. Moreover, the effect of dose differences in OADs on the therapeutic effect was not taken into account owing to the high level of inconsistency when the analysis was stratified by dosage. Thus, when they are applied clinically, the specific conditions of individual patients should be taken into account. Second, only a small number of studies reported MACEs (including zero events), which may have reduced the statistical robustness when calculating MACEs. Given the limited data available, the RCTs included for patients with MACEs were relatively broad, involving cardiovascular death, nonfatal myocardial infarction, and nonfatal stroke; although this approach was more comprehensive, it increased the heterogeneity. Third, the numbers of participants in some treatment arms (such as SGLT2i/Met versus DPP-4i/Met, and DPP-4i/Met versus AGI/Met) were vastly different for $2 \mathrm{~h}-\mathrm{PPG}$, which may limit the statistical power (increased potential for type 2 error). Further studies with a larger sample size and more head-to-head comparisons are required to substantiate the present results. Fourth, data sources in this study were mainly concentrated in the white population, and there was a relatively small sample of the Asian population. It is expected that there will be more clinical data from the Asian population in the future. Fifth, we did not assess the baseline age, baseline diabetes duration, duration of treatment, and duration of follow-up as effect modifiers on estimates for efficacy and safety outcomes; the focus of further studies should be on evaluating the effects of these variables. Finally, only one study that involved the addition of meglitinides to metformin for T2DM treatment was included in our study; this was accompanied by a relatively wide CI, which in turn resulted in a relatively low statistical robustness in the final results. Our results are limited in this regard. More data are needed to support our findings.

\section{CONCLUSION}

Our results suggest that the seven classes of OADs were not associated with any increased risk of MACEs or all-cause mortality when added to metformin. Most OADs were 
associated with similarly large reductions in HbA1c levels when added to metformin, while SGLT-2 inhibitors as add-ons to metformin might be the best option when considering factors including body weight, FPG, and 2-h PPG. As the evidence for cardiovascular safety in dual therapy with metformin is extremely limited, further evidence is awaited to confirm the findings of this analysis.

\section{ACKNOWLEDGEMENTS}

Funding. No funding or sponsorship was received for this study or publication of this article. The article processing charges were funded by the authors.

Editorial Assistance. We thank Editage for carrying out English language editing of the manuscript; the polishing charges were funded by the authors.

Authorship. All named authors meet the International Committee of Medical Journal Editors (ICMJE) criteria for authorship for this article, take responsibility for the integrity of the work as a whole, and have given their approval for this version to be published.

Disclosures. Dan Qian, Tiantian Zhang, Peiying Zheng, Zhuoru Liang, Sen Wang, Jingmei Xie, Lina Zhao, Ying Zhang, and Bing Situ declare they have no conflict of interest to disclose.

Compliance with Ethics Guidelines. This article is based on previously conducted studies and does not contain any studies with human participants or animals performed by any of the authors.

Data Availability. The datasets generated during and/or analyzed during the current study are available from the corresponding author on reasonable request.

Open Access. This article is distributed under the terms of the Creative Commons
Attribution-NonCommercial 4.0 International License (http://creativecommons.org/licenses/ by-nc/4.0/), which permits any noncommercial use, distribution, and reproduction in any medium, provided you give appropriate credit to the original author(s) and the source, provide a link to the Creative Commons license, and indicate if changes were made.

\section{REFERENCES}

1. Defronzo RA. Banting Lecture. From the triumvirate to the ominous octet: a new paradigm for the treatment of type 2 diabetes mellitus. Diabetes. 2009;58:773-95.

2. Prasad R, Groop L. Genetics of type 2 diabetespitfalls and possibilities. Genes (Basel). 2015;6:87-123.

3. International Diabetes Federation. IDF diabetes atlas, 8th edition. http://www.diabetesatlas.org/ across-the-globe.html. Accessed 15 Nov 2017.

4. Inzucchi SE, Bergenstal RM, Buse JB, et al. Management of hyperglycemia in type 2 diabetes, 2015: a patient-centered approach: update to a position statement of the American Diabetes Association and the European Association for the Study of Diabetes. Diabetes Care. 2015;382015:140-9.

5. Eizirik DL, et al. Medical management of hyperglycemia in type 2 diabetes: a consensus algorithm for the initiation and adjustment of therapy: a consensus statement of the American Diabetes Association and the European Association for the Study of Diabetes: response to Nathan et al. Diabetes Care. 2009;32:e35-6.

6. International Diabetes Federation Guideline Development Group. Global guideline for type 2 diabetes. Diabetes Res Clin Pract. 2014;104:1-52.

7. Ganesan P, Arulselvan P, Choi D. Phytobioactive compound-based nanodelivery systems for the treatment of type 2 diabetes mellitus-current status. Int J Nanomed. 2017;12:1097-111.

8. Li J, Gong Y, Li C, Lu Y, Liu Y, Shao Y. Long-term efficacy and safety of sodium-glucose cotransporter2 inhibitors as add-on to metformin treatment in the management of type 2 diabetes mellitus. Medicine (Baltimore). 2017;96:e7201.

9. Jansen JP, Trikalinos T, Cappelleri JC, et al. Indirect treatment comparison/network meta-analysis study questionnaire to assess relevance and credibility to 
inform health care decision making: an ISPORAMCP-NPC Good Practice Task Force report. Value Health. 2014;17:157-73.

10. Higgins J, Altman D, Gotzsche $P$, et al. The Cochrane Collaboration's tool for assessing risk of bias in randomised trials. BMJ. 2011;343:d5928.

11. DerSimonian R, Laird N. Meta-analysis in clinical trials revisited. Contemp Clin Trials. 2015;45:139-45.

12. Guyatt G, Oxman A, Kunz R, et al. GRADE guidelines: 7. Rating the quality of evidence-inconsistency. J Clin Epidemiol. 2011;64:1294-302.

13. Lu G, Ades A. Combination of direct and indirect evidence in mixed treatment comparisons. Stat Med. 2004;23:3105-24.

14. Chaimani A, Higgins J, Mavridis D, Spyridonos P, Salanti G. Graphical tools for network meta-analysis in STATA. PLoS One. 2013;8:e76654.

15. White I, Barrett J, Jackson D, Higgins JP. Consistency and inconsistency in network meta-analysis: model estimation using multivariate meta-regression. Res Synth Methods. 2012;3:111-25.

16. Salanti G. Indirect and mixed-treatment comparison, network, or multiple-treatments meta-analysis: many names, many benefits, many concerns for the next generation evidence synthesis tool. Res Synth Methods. 2012;3:80-97.

17. Salanti G, Ades A, Ioannidis J. Graphical methods and numerical summaries for presenting results from multiple-treatment meta-analysis: an overview and tutorial. J Clin Epidemiol. 2011;64:163-71.

18. Keus F, Wetterslev J, Gluud C, Gooszen HG, van Laarhoven CJ. Robustness assessments are needed to reduce bias in meta-analyses that include zeroevent randomized trials. Am J Gastroenterol. 2009;104:546-51.

19. Veroniki A, Vasiliadis H, Higgins J, Salanti G. Evaluation of inconsistency in networks of interventions. Int J Epidemiol. 2013;42:919.

20. Song F. Validity of indirect comparison for estimating efficacy of competing interventions: empirical evidence from published meta-analyses. BMJ. 2003;326:472.

21. Higgins J, Jackson D, Barrett J, Lu G, Ades A, White I. Consistency and inconsistency in network metaanalysis: concepts and models for multi-arm studies. Res Synth Methods. 2012;3:98-110.

22. Egger M, Smith G, Schneider M, Minder C. Bias in meta-analysis detected by a simple, graphical test. BMJ. 1997;315:629-34.
23. Barnett K, Ogston S, McMurdo M, Morris A, Evans J. A 12-year follow-up study of all-cause and cardiovascular mortality among 10,532 people newly diagnosed with type 2 diabetes in Tayside, Scotland. Diabet Med. 2010;27:1124-9.

24. Vaccaro O. Impact of diabetes and previous myocardial infarction on long-term survival: 25-year mortality follow-up of primary screenees of the multiple risk factor intervention trial. Arch Intern Med. 2004;164:1433-8.

25. Palmer SC, Mavridis D, Nicolucci A, et al. Comparison of clinical outcomes and adverse events associated with glucose-lowering drugs in patients with type 2 diabetes. JAMA. 2016;316:313.

26. Wu JHY, Foote C, Blomster J, et al. Effects of sodium-glucose cotransporter- 2 inhibitors on cardiovascular events, death, and major safety outcomes in adults with type 2 diabetes: a systematic review and meta-analysis. Lancet Diabetes Endocrinol. 2016;4:411-9.

27. Saad M, Mahmoud AN, Elgendy IY, et al. Cardiovascular outcomes with sodium-glucose cotransporter-2 inhibitors in patients with type II diabetes mellitus: a meta-analysis of placebo-controlled randomized trials. Int J Cardiol. 2017;228:352-8.

28. Zinman B, Wanner C, Lachin JM, et al. Empagliflozin, cardiovascular outcomes, and mortality in type 2 diabetes. N Engl J Med. 2015;373:2117-28.

29. Tang H, Fang Z, Wang T, Cui W, Zhai S, Song Y. Meta-analysis of effects of sodium-glucose cotransporter 2 inhibitors on cardiovascular outcomes and all-cause mortality among patients with type 2 diabetes mellitus. Am J Cardiol. 2016;118:1774-80.

30. American Diabetes Association. 8. Pharmacologic approaches to glycemic treatment. Diabetes Care. 2017;40:S64-74.

31. Neal B, Perkovic V, Mahaffey KW, et al. Canagliflozin and cardiovascular and renal events in type 2 diabetes. N Engl J Med. 2017;377:644-57.

32. NLM. ClinicalTrials.gov: CANVAS_CANagliflozin cardioVascular Assessment Study (CANVAS). https://clinicaltrials.gov/ct2/show/NCT01032629. Accessed 25 Oct 2017.

33. NLM. ClinicalTrials.gov: Multicenter Trial to Evaluate the Effect of Dapagliflozin on the Incidence of Cardiovascular Events (DECLARE-TIMI58). https:// clinicaltrials.gov/ct2/show/NCT01730534. Accessed 16 Oct 2017.

34. NLM. ClinicalTrials.gov: Cardiovascular Outcomes Following Ertugliflozin Treatment in Type 2 Diabetes Mellitus Participants With Vascular Disease, 
The VERTIS CV Study (MK-8835-004). https:// clinicaltrials.gov/ct2/show/NCT01986881. Accessed 5 Nov 2017.

35. Drucker DJ, Nauck MA. The incretin system: glucagon-like peptide-1 receptor agonists and dipeptidyl peptidase- 4 inhibitors in type 2 diabetes. Lancet. 2006;368:1696-705.

36. Holst JJ, Windelov JA, Boer GA. Searching for the physiological role of glucose-dependent insulinotropic polypeptide. J Diabetes Investig. 2016;7(Suppl Suppl 1):8-12.

37. Yanagimachi T, Fujita Y, Takeda Y, et al. Dipeptidyl peptidase-4 inhibitor treatment induces a greater increase in plasma levels of bioactive GIP than GLP1 in non-diabetic subjects. Mol Metab. 2017;6:226-31.

38. Sharma A, Paliwal G, Upadhyay N, Tiwari A. Therapeutic stimulation of GLP-1 and GIP protein with DPP-4 inhibitors for type-2 diabetes treatment. J Diabetes Metab Disord. 2015;14:15.

39. Zhang Z, Chen X, Lu P, et al. Incretin-based agents in type 2 diabetic patients at cardiovascular risk: compare the effect of GLP-1 agonists and DPP-4 inhibitors on cardiovascular and pancreatic outcomes. Cardiovasc Diabetol. 2017;16:31.

40. Wang W, Zhou X, Kwong JSW, et al. Efficacy and safety of thiazolidinediones in diabetes patients with renal impairment: a systematic review and meta-analysis. Sci Rep. 2017;7:1717.

41. Lincoff AM, Wolski K, Nicholls SJ, Nissenal SE. Pioglitazone and risk of cardiovascular events in patients with type 2 diabetes mellitus: a metaanalysis of randomized trials. JAMA. 2007;298:1180-8.
42. Singh S, Loke YK, Furberg CD. Long-term risk of cardiovascular events with rosiglitazone: a metaanalysis. JAMA. 2007;298:1189-95.

43. White WB, Cannon CP, Heller SR, et al. Alogliptin after acute coronary syndrome in patients with type 2 diabetes. N Engl J Med. 2013;369:1327-35.

44. Vaccaro O, Masulli M, Nicolucci A. Effects on the incidence of cardiovascular events of the addition of pioglitazone versus sulfonylureas in patients with type 2 diabetes inadequately controlled with metformin (TOSCA.IT): a randomised, multicentre trial. Lancet Diabetes Endocrinol. 2007;5:887-97.

45. NLM. ClinicalTrials.gov: Cardiovascular Outcomes in Participants with Type 2 Diabetes Mellitus (T2DM). https://clinicaltrials.gov/ct2/show/NCT03249 506. Accessed 27 Oct 2017.

46. Zhang R, Reisin E. Obesity-hypertension: the effects on cardiovascular and renal systems. Am J Hypertens. 2000;13:1308-14.

47. Eeg-Olofsson K, Cederholm J, Nilsson PM, et al. Risk of cardiovascular disease and mortality in overweight and obese patients with type 2 diabetes: an observational study in 13,087 patients. Diabetologia. 2009;52:65-73.

48. Fujioka K, Seaton TB, Rowe E, et al. Weight loss with sibutramine improves glycaemic control and other metabolic parameters in obese patients with type 2 diabetes mellitus. Diabetes Obes Metab. 2000;2:175-87.

49. Zaccardi F, Webb DR, Htike ZZ, Youssef D, Khunti $\mathrm{K}$, Davies MJ. Efficacy and safety of sodium-glucose co-transporter-2 inhibitors in type 2 diabetes mellitus: systematic review and network meta-analysis. Diabetes Obes Metab. 2016;18:783-94. 